
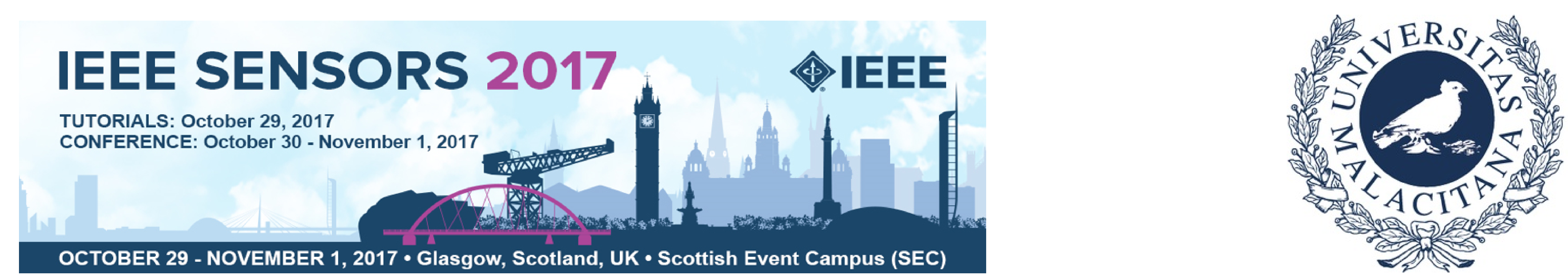

\title{
Human and Object Recognition with a High-Resolution Tactile Sensor
}

\author{
Juan M. Gandarias, Jesús M. Gómez-de-Gabriel and Alfonso J. García-Cerezo \\ System Engineering and Automation Department, University of Málaga \\ jmgandarias@uma.es
}

\section{Introduction}

Touch sense is critical for humans. We use the sense of touch to perform complexes tasks such as recognising objects. To perform this task, human beings need two abilities: first, being able to extract information through touch, and second, having cognitive capabilities to process this information. As current trends in robotics are focusing on providing intelligence to robots and making them more similar to humans, tactile sensing in field robotics, is a key problem. The resurgence of artificial intelligence $(\mathrm{Al})$ methods is a great help for interpreting the information acquired. Recent applications propose the use of a tactile sensor to extract information from the object touched and a learning process that use this information to distinguish familiar objects among the collected data.

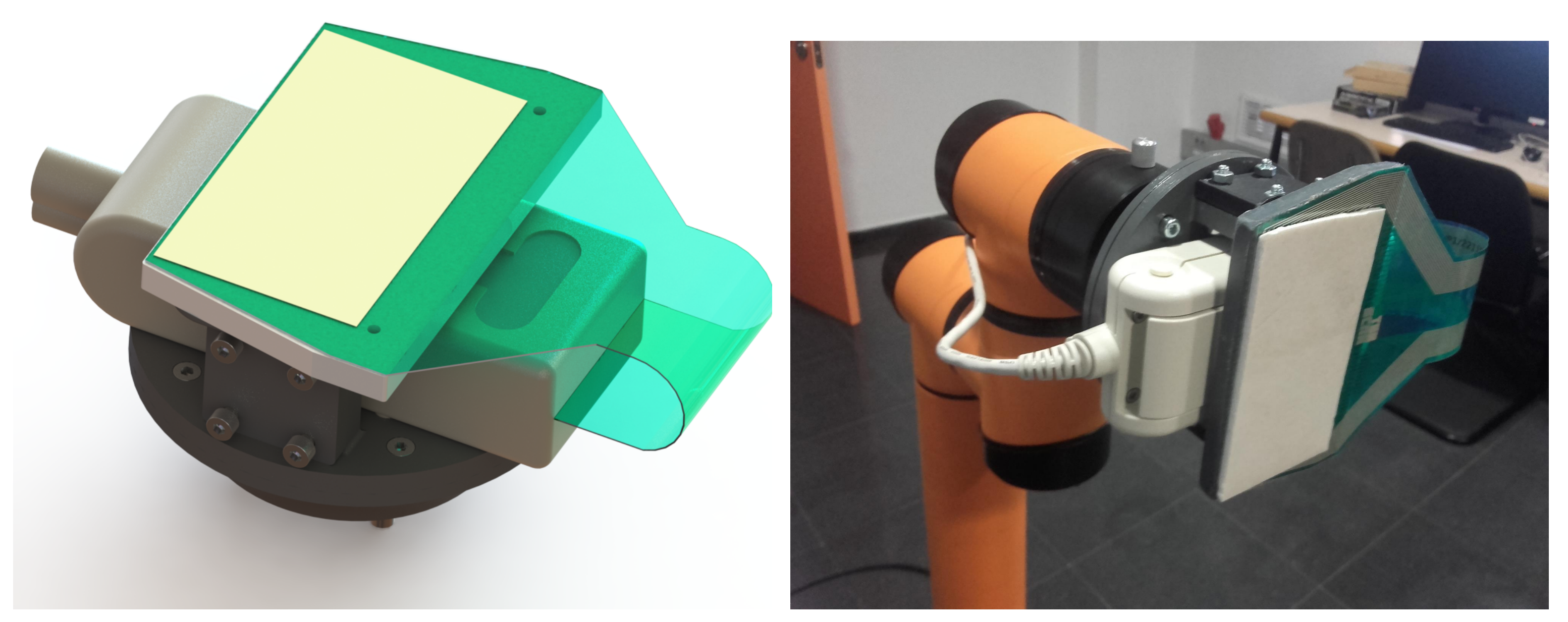

Two methods for recognising objects through pressure images are presented. The first method uses the Speeded-Up Robust Features (SURF) as a feature extractor, whilst the second method uses the Deep Convolutional Neural Network (AlexNet). Then, both methods include a Supported Vector Machine (SVM) to get a classifier. Furthermore we compare both methods results in terms of accuracy and computation time. On the other hand, we propose a real application of tactile sensors and object recognition to the field of rescue robotics. An experiment for testing the performance of the methods is carried out under controller conditions.

\section{Methods}

- SURF + SVM: This method needs to include an intermediate step between the features extraction and the supervised learning. A $k$-means unsupervised algorithm is implemented to cluster features into a dictionary, generating a framework of Bag of Words (BoW). Then, a supervised SVM is trained to generate a classifier.

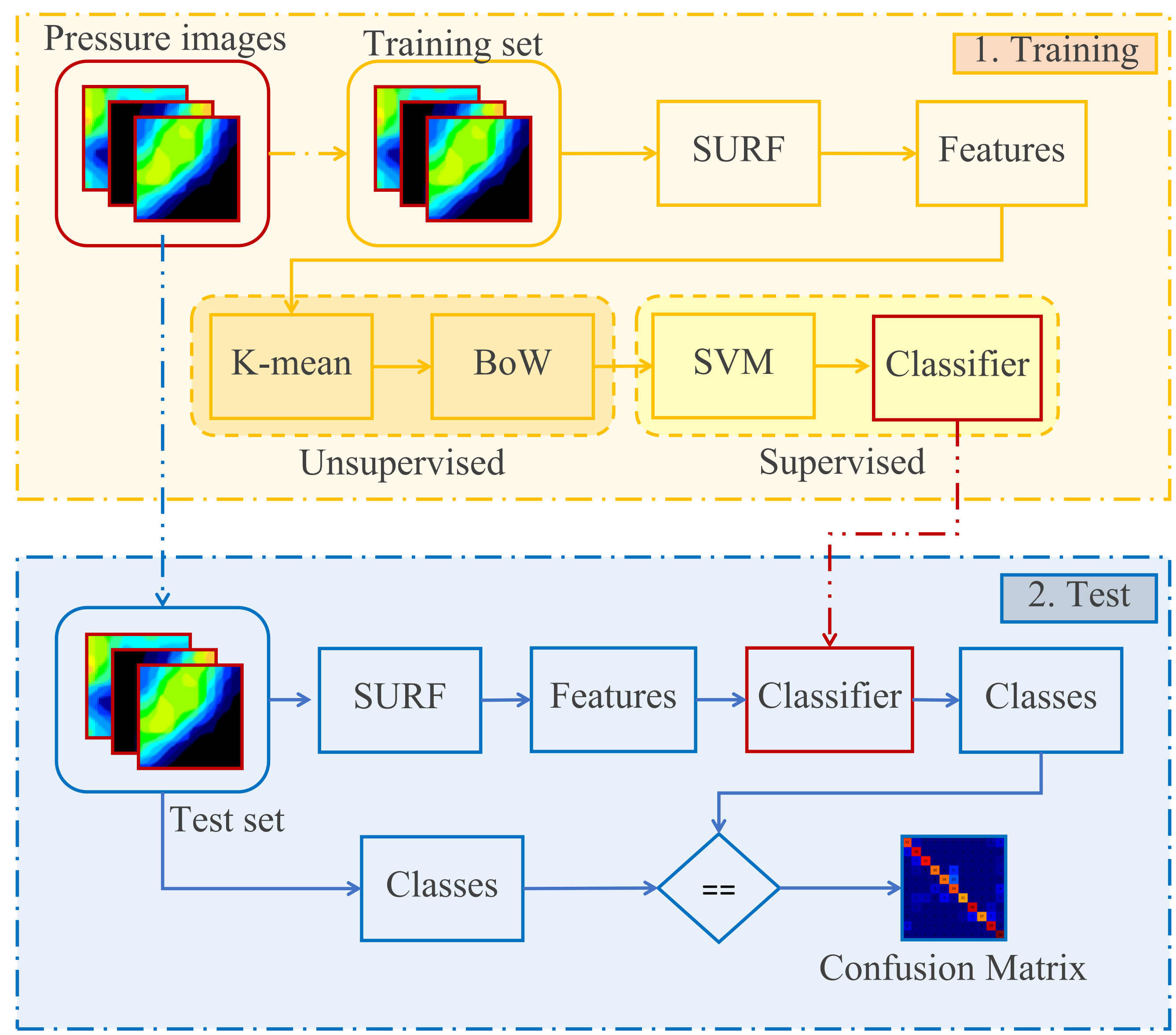

- DCNN + SVM: The second method implements a DCNN to extract features. This procedure consists of using a pre-trained network to classify conventional images taking with a camera. Activations of the last layer before the classification are used to describe features. After that, a supervised $S V M$ is trained to get a classifier.

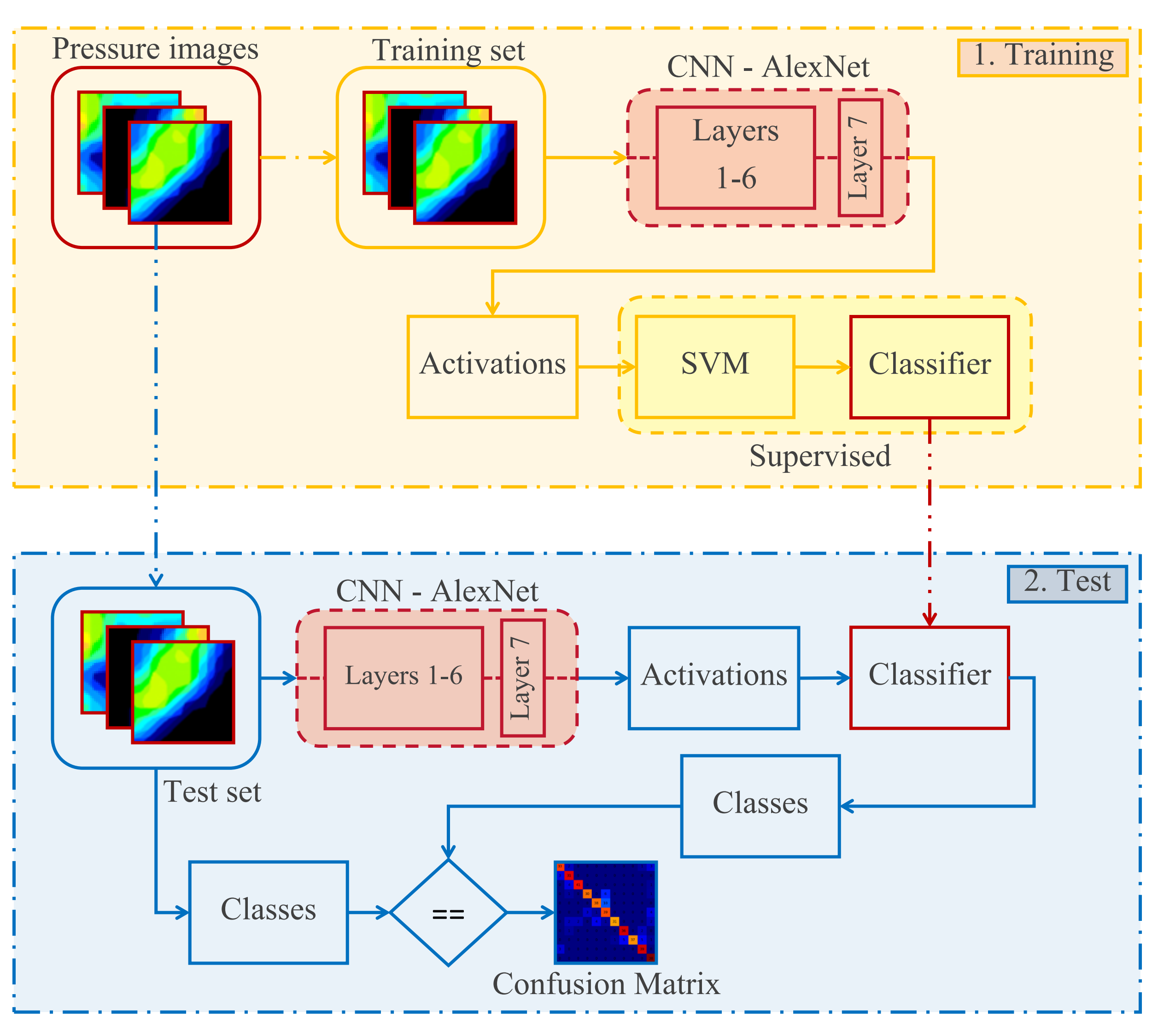

Experiments and Results

- Experimental setup:

A high-resolution tactile sensor has been attached to the 6 DOF robotic arm AUBO Our-i5. The Tekscan pressure mapping sensor 6077 is conformed by 1400 resistive sensels of pressure distributed on 28 rows and 50 columns with a size of $53.3 \mathrm{~mm} \times 95.3 \mathrm{~mm}$.

D Dataset:

A total of 400 pressure images have been used to feed each method. These images are divided into eight classes labelled as: Finger, Hand, Arm, Pen, Scissors, Pliers, Sticky Tape, and Allen Key. The training set is composed by 160 images, 20 images for each label, whilst the test set is composed by 240, 30 images for each label.

- Results:
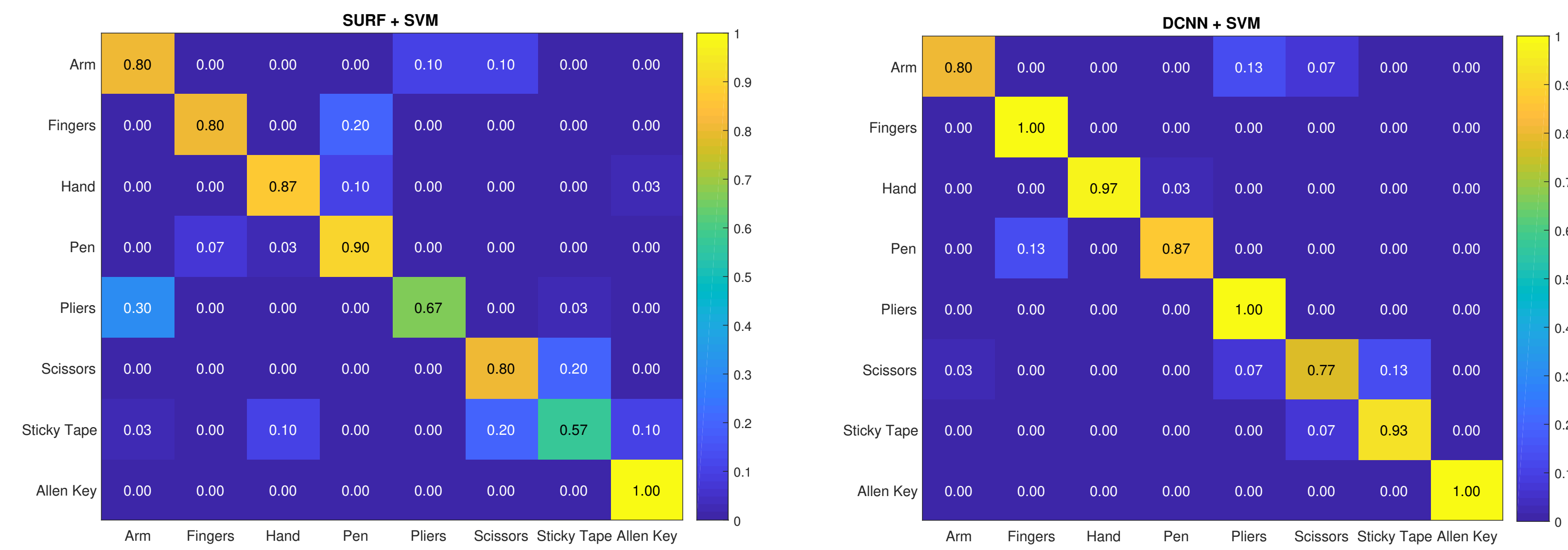

\begin{tabular}{c|c|c|c} 
Method & Accuracy (\%) & Improvement (\%) & Time (s) \\
\hline SURF + SVM & 80 & - & 0.01 \\
$D C N N+$ SVM & 91.67 & 11.67 & 0.7
\end{tabular}

\section{Conclusions and Future Work}

- Conclusions:

DCNN-based method has achieved an $11.67 \%$ improvement with respect to the method 1 , however, the computational time was $0.7 \mathrm{~s}$ in the method 2 opposite to $0.01 \mathrm{~s}$ in the method 1 . Although the computation time provided shall not be a conclusive evidence, it sheds light on the computational load ratio between methods.

- Future work:

We aim to compare our results with existing solutions, and to take advantages of using combined tactile and kinesthetic information. Also, other sensors will be integrated to extract additional information of the state of the victim.

\section{Ackowledgment}

This work was partially supported by the Spanish project DPI2015-65186-R and the European Commission under grant agreement BES-2016-078237. 